\section{Setting a trap for cytokines}

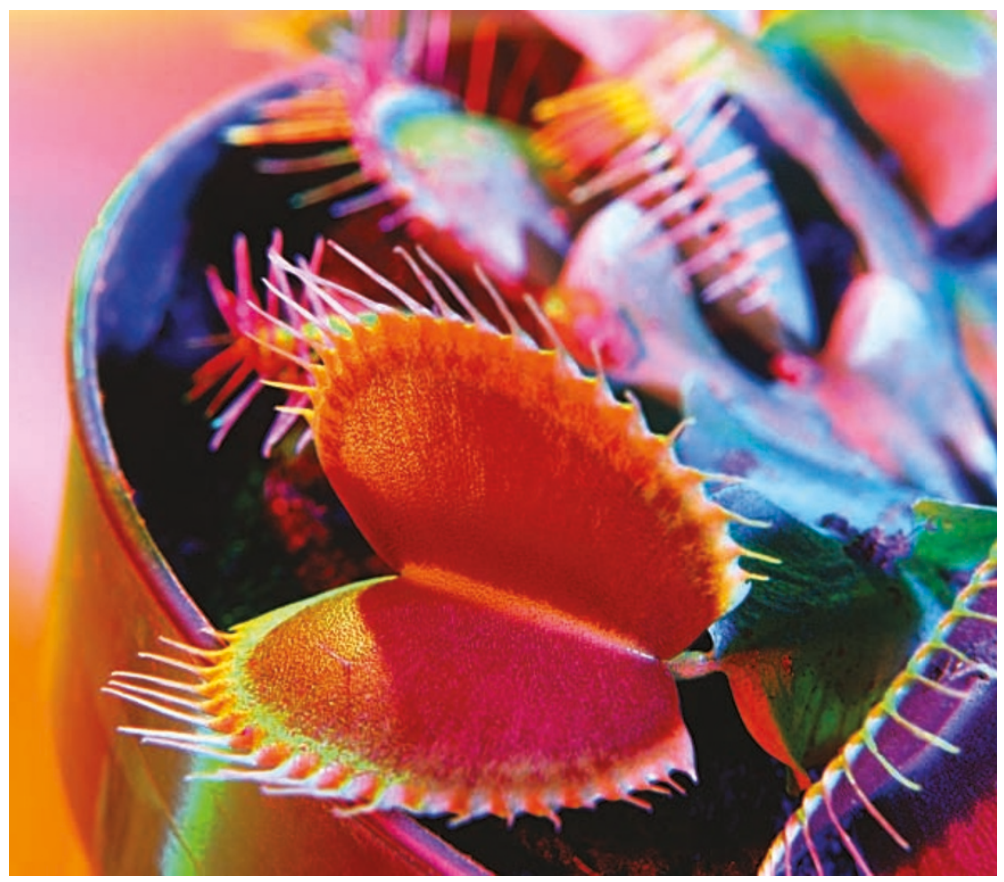

Considerable advances in treating autoimmune diseases have been made with agents that block the action of cytokines. New work published in the January issue of Nature Medicine describes the design and production of a novel class of cytokine antagonists, termed cytokine traps, which overcome some of the problems with existing antagonists.

The best results to date with cytokine antagonists have been achieved using soluble decoy receptors that bind and block the cytokine of interest. The tumour necrosis factor- $\alpha$ (TNF- $\alpha$ ) blocker etanercept (Enbrel), which is made up of the extracellular ligand-binding portion of the TNF- $\alpha$ receptor fused to the constant region $(\mathrm{Fc})$ of a human immunoglobulin (Ig) chain, has been approved for the treatment of rheumatoid arthritis (RA), and has good clinical activity. However, for most cytokines, two distinct receptor components cooperate to bind the cytokine very tightly, and so a single-component receptor antagonist does not work very well. Perhaps because of these limitations, single-component soluble receptor antagonists for IL- 1 and IL-4 have not been successful.

In an effort to overcome problems of low binding affinity, and short half-life (which necessitates frequent injections), Stahl and colleagues developed soluble cytokine receptors, termed traps, that incorporate both of the receptor components normally required to achieve high-affinity binding. The traps were constructed by engineering linear fusions of both receptor extracellular domains followed by the human IgG1 Fc domain. The Fc portion directed the formation of disulphide-linked dimers. The affinity of cytokines for the traps is significantly greater than for their individual cellular receptor components. IL-1, IL-4 and IL-6 traps were able to

\section{Fast-track to a male pill?}

None of the available options for male contraception, such as condoms, vasectomy and withdrawal, offer the ideal combination of convenience, effectiveness and reversibility. And although considerable efforts have been directed at developing a reversible oral contraceptive drug for men, those drugs closest to the market, which target hormones such as testosterone that are involved in the production of sperm, can cause side effects including weight gain. Now, findings by van der Spoel et al., reported in the Proceedings of the National Academy of Sciences, indicate that treatment with a drug recently approved for Gaucher's disease, a rare genetic disorder, might also be a convenient non-hormonal approach to reversible male contraception.
Male mice orally dosed with the drug the alkylated iminosugar $N$-butyldeoxynojirimycin (NB-DNJ) - became sterile after three weeks of treatment, whereas female mice were unaffected. Drug treatment did not influence levels of testosterone, or levels of the reproductive hormones luteinizing hormone and follicle-stimulating hormone, indicating that $N \mathrm{~B}-\mathrm{DNJ}$ acts in a non-hormonal manner. And once taken off the drug, male mice regained their fertility after three weeks, and their resultant offspring developed normally.

Sperm from drug-treated mice show several morphological abnormalities, and have severely impaired motility. But how might the drug work at the molecular level? $\mathrm{NB}-\mathrm{DNJ}$ is a well-characterized inhibitor of ceramide-specific glucosyltransferase, a key enzyme involved in the production of glucosphingolipids (GSLs) present on the membrane of all mammalian cells. It is this activity that gives NB-DNJ efficacy in Gaucher's disease, which is caused by a build-up of GSLs owing to defects in an enzyme involved in GSL degradation - by reducing GSL synthesis, NB-DNJ acts to restore the proper balance. GSLs are also important for sperm production, as mice deficient in enzymes involved in GSL biosynthesis are sterile, leading the authors to suggest that the impact of NB-DNJ on male fertility might be caused by altering GSL metabolism during sperm development.

Given the highly conserved nature of sperm production in mammals, it seems plausible that NB-DNJ could have analogous effects in man. And because NBDNJ has already been extensively studied in the clinic, it could be rapidly evaluated for its suitability as a male contraceptive. In trials so far, the major side effect of NB-DNJ has been dose-dependent diarrhea, but if the very low doses needed to cause infertility in mice were reflected in humans, such side effects might be negligible.

(2) References and links Peter Kirkpatrick ORIGINAL RESEARCH PAPER van der Spoel, A. C. et al. Reversible infertility in male mice after oral administration of alkylated imino sugars: A nonhormonal approach to male contraception. Proc. Natt Acad. Sci. USA 26, 17173-17178 (2002)

FURTHER READING Dwek, R. A. et al. Targeting glycosylation as a therapeutic approach. Nature Rev. Drug Discov. 1, 65-75 (2002) 
bind the specific cytokine in vitro with high affinity and potently block cytokine action. In vivo, mouse versions of the IL- 1 and IL-4 traps were able to block the development of arthritic joints in a mouse arthritis model and to prevent the high numbers of eosinophils that accumulate in mouse models of asthma, respectively. The human IL-4 trap was also able to block IL-4 action in primates.

Phase II human clinical trials of the IL- 1 trap to assess safety and efficacy in RA patients are underway. Pharmacokinetic data suggest that dosing is compatible with a onceweekly shot, which would be a great improvement over existing treatments, which are injected daily.

Melanie Brazil

(2) References and links ORIGINAL RESEARCH PAPER Economides, A. N. et al. Cytokine traps: multicomponent high affinity blockers of cytokine action. Nature Med. 9, 47-52 (2003) FURTHER READING Dinarello, C. A. Setting the cytokine trap for autoimmunity. Nature Med. 9, 20-22 (2003) | Stahl, N. \& Yancopoulos, G. D. The alphas, betas and kinases of cytokine receptor complexes. Cell 74, 587-590 (1993)

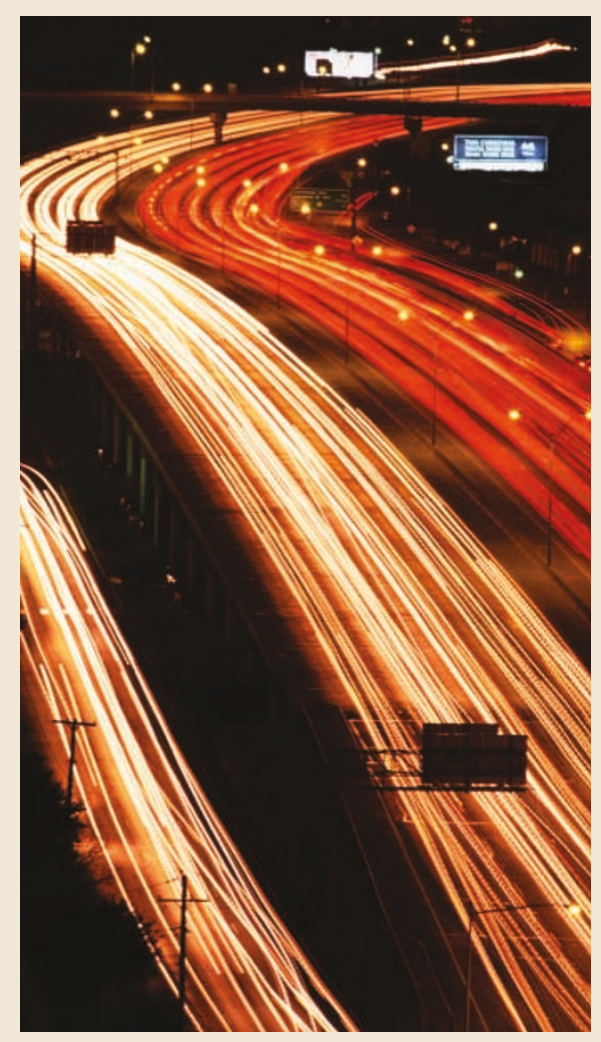

ANTIHYPERTENSIVE DRUGS

\section{ALLHATs off to the golden oldie}

"Quite simply, the Antihypertensive and LipidLowering Treatment to Prevent Heart Attack Trial (ALLHAT) is one of the most important trials of antihypertensive therapy", states Lawrence Appel in the Journal of the American Medical Association, in response to the eagerly awaited results from the biggest head-to-head comparison to find the most effective class of hypertension drug. And the results from the trial are striking the oldest form of treatment seems to be more effective than the newer kids on the block.

Diuretics have been used to treat high blood pressure since the late 1950s. But in the past couple of decades, several classes of treatments with different mechanisms of action have emerged, such as calcium channel blockers (CCBs) and angiotensin-converting enzyme (ACE) inhibitors. These newer treatments have theoretical advantages over diuretics, but with little practical proof of their benefits clinicians have debated which classes of treatment are more likely to work and should therefore be prescribed first to patients.

So, in 1994 a randomized, double-blind trial called ALLHAT, supported by the US National Heart, Lung and Blood Institute, was launched to compare the outcomes of a CCB (amlodipine) and an ACE inhibitor (lisinopril) with a diuretic (chlorthalidone) in over 33,000 hypertensive patients. (Another class of antihypertensive, an $\alpha$-blocker (doxazosin) was also compared, but was withdrawn during the trial because "it was found to be inferior to the diuretic".) After following the patients for around 5 years, the researchers found that the diuretic was just as effective in preventing the primary end point (the incidence of fatal coronary heart disease (CHD) or nonfatal heart attack) as the other drugs. But in the prevention of secondary outcomes (all-cause mortality, stroke, combined CHD or combined cardiovascular disease), better results and a lower cost gave the diuretic the edge over its counterparts.

The findings from the ALLHAT study will have a great impact on medical care and research. As the study looked at a broad range of people with hypertension, its findings imply that diuretics will be a more appropriate choice over ACE inhibitors and CCBs in almost all cases. And as diuretics cost around 6-20 times less per pill than the other classes, prescribing them first would dramatically reduce healthcare costs (the annual US antihypertensive drug cost is $\sim \$ 15$ billion).

Also, the finding that newer drugs do not necessarily mean better drugs will re-ignite arguments surrounding the approval process.

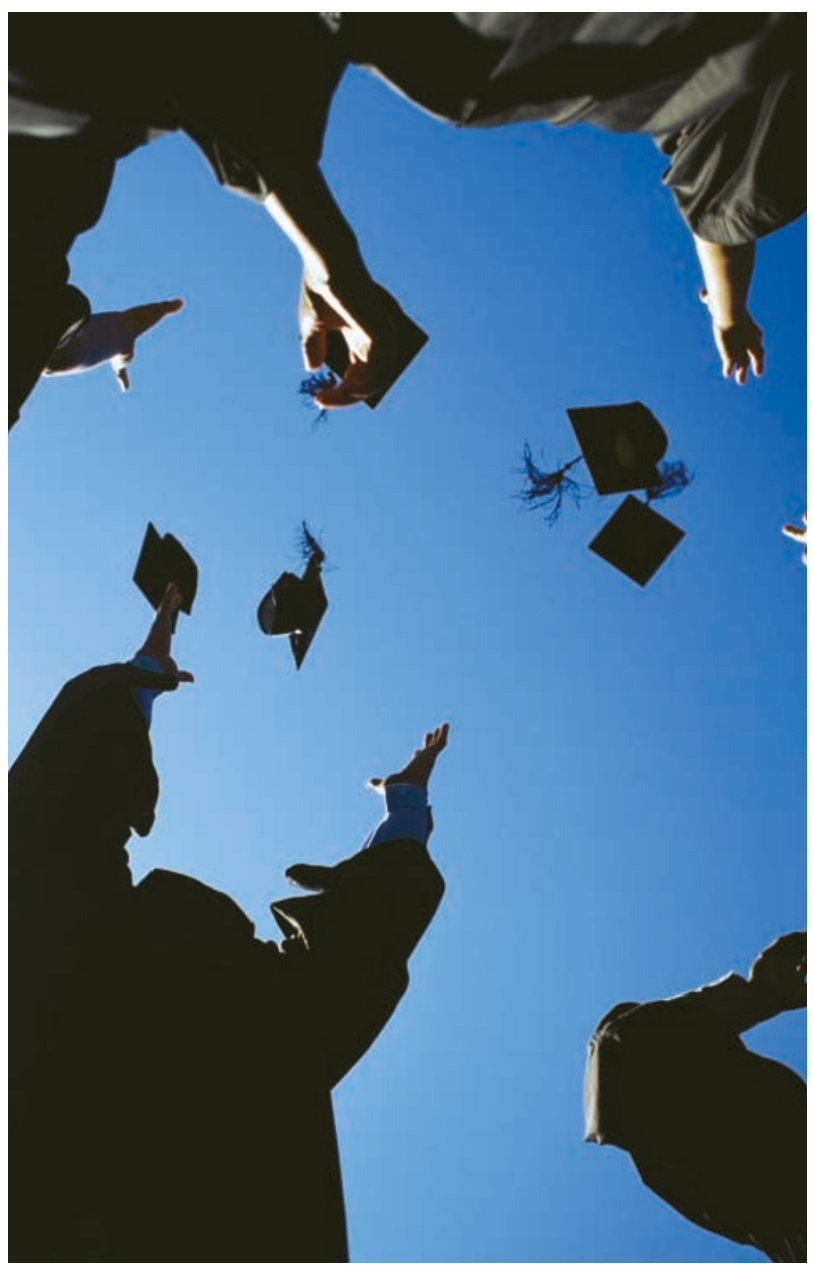

Approval of a drug only requires proof of efficacy compared with placebo, not whether it works better than existing therapies. In a year that saw another US-government-funded trial disprove the theory that hormone-replacement therapy protects women against heart disease, the findings from the ALLHAT study raise the question of whether one role of governments should be to fund more studies that look into the important medical and economic issues that industry-based trials do not typically address.

Simon Frantz

(4) References and links ORIGINAL RESEARCH PAPER The ALLHAT Officers and

Coordinators for the ALLHAT Collaborative Research Group. Major outcomes in high-risk hypertensive patients randomized to angiotensin-converting enzyme inhibitor or calcium channel blocker vs diuretic: the Antihypertensive and Lipid-Lowering Treatment to Prevent Heart Attack Trial (ALLHAT). JAMA 288, 2981-2997 (2002) FURTHER READING Zaman, M. A., Oparil, S.

\& Calhoun, D. A. Drugs targeting the renin-angiotensin-aldosterone system. Nature Rev. Drug Disc. 1, 621-636 (2002) 